\title{
Die „neue“ Borderline-Persönlichkeitsstörung: Dimensionale Klassifikation im DSM-5 und ICD-11
}

\author{
Horst Mitmansgruber
}

Online publiziert: 14 . Oktober 2020

(c) Der/die Autor(en) 2020

Zusammenfassung Die jahrzehntelange und zunehmende Unzufriedenheit mit der bisherigen kategorialen Klassifikation der Persönlichkeitsstörungen (PS) im amerikanischen DSM-IV und in der ICD-10 der Weltgesundheitsorganisation hat $u$.a. mit fehlender empirischer Unterstützung vieler Kategorien, der sehr hohen Komorbidität der PS untereinander oder der großen Heterogenität von Symptomen innerhalb einer Diagnose zu tun. Sie hat in den letzten Revisionen der beiden Diagnosesysteme einen radikalen Wandel hin zu einem dimensionalen Klassifikationssystem unterstützt, das um vieles stärker in der empirischen psychologischen Forschung abgesichert ist. Im DSM-5 ist die Revolution ausgeblieben, weil das dimensionale Modell im Anhang verblieben ist, während die alte DSM-IV-Klassifikation unverändert übernommen wurde. Allerdings hat dieses „Alternative Modell der Persönlichkeitsstörungen“ (AMPD) seit seiner Publikation erhebliches Forschungsinteresse erfahren. In der ICD-11 ist eine dimensionale Einschätzung der Persönlichkeit auf den 5 Domänen „Negative Affectivity“, „Detachment“, „Dissociality“, „Disinhibition“ und „Anankastia“ als radikale Alternative zu den 10 bisherigen Kategorien bereits abgesegnet und wird ab 2022 weltweit die Diagnosestellung verändern. Während hier zunächst alle bisherigen Kategorien eliminiert wurden, erreichten kritische Stimmen am Ende, dass die Borderline-Störung als einziger Qualifier in der ICD-11 erhalten bleibt. Die beiden Systeme werden in

\footnotetext{
H. Mitmansgruber ( $\bowtie)$

Universitätsklinik für Medizinische Psychologie,

Medizinische Universität Innsbruck,

Speckbacherstraße 23, 6020 Innsbruck, Österreich

Arbeitsgemeinschaft für Verhaltensmodifikation -

Österreich (AVM), Paris-Lodron-Straße 32, 5020 Salzburg,

Österreich

horst.mitmansgruber@i-med.ac.at
}

ihren zentralen Annahmen und im praktischen Vorgehen beleuchtet. Die Diskussionen und die bisherige empirische Befundlage zu den Dimensionen und der klinischen Nützlichkeit für Praktiker*innen werden zusammengefasst.

Schlüsselwörter Borderline-Persönlichkeitsstörung · Dimensionale Klassifikation der

Persönlichkeitsstörungen · ICD-11 · DSM-5 ·

Alternatives Modell der Persönlichkeitsstörungen (AMPD)

The "new" borderline personality disorder: Dimensional classification in DSM-5 and ICD-11

Summary Decades of increasing discontent with current categorical classification systems of personality disorders (PD) in the American DSM-IV and the ICD10 of the World Health Organization are based on missing empirical support of many of the categories, high comorbidity of PDs and large heterogeneity of symptoms within a diagnosis. This discontent has paved the way for a radical change in the last revisions of these diagnostic systems to replace a categorical with a dimensional model of classification, which is strongly supported by empirical psychological research. For the DSM-5 the revolution was cancelled, as the dimensional model was placed in Section III "Emerging models" whereas the old DSM-IV categorical system was adopted without any changes. However, this "Alternative Model of Personality Disorders" (AMPD) has received much attention in research since its publication. In the ICD-11, a dimensional assessment of personality on five domains ("Negative Affectivity", "Detachment", "Dissociality", "Disinhibition" and "Anankastia") as a radical alternative to currently existing 10 categories is officially accepted and will change diagnosis of personality disorders world- 
wide from 2022. Whereas all current categories have been eliminated in the proposal, at first, critics were able to reclaim a "borderline pattern" as the one and only qualifier for a specific category in the final version of the ICD-11. Both systems are illustrated in their central assumptions and practical procedures. Discussions on dimensions, their current empirical basis and clinical utility are summarized.

Keywords Borderline personality disorder . Dimensional classification of personality disorders . ICD-11 · DSM-5 - Alternative Model of Personality Disorders (AMPD)

\section{Die Borderline-Persönlichkeitsstörung im DSM- IV und ICD-10: Probleme mit dem kategorialen Diagnosesystem}

Die kategoriale Diagnosestellung der Persönlichkeitsstörungen (PS) in den aktuell gültigen Diagnosesystemen ist uns über die vergangenen bald drei Jahrzehnte schon sehr vertraut geworden. Die aktuell noch gültige ICD-10 der Weltgesundheitsorganisation (World Health Organization 1992) und das DSM-IV bzw. DSM-5 ${ }^{1}$ der amerikanischen Psychiatrie (American Psychiatric Association 1994, 2013) beschreiben neun bzw. zehn spezifische PS-Diagnosen. Sie werden diagnostiziert, wenn allgemeine Kriterien einer PS (merklich abweichendes, zeitlich überdauerndes Muster von Kognitionen, Affekten, zwischenmenschlichem Verhalten oder Impulsivität, das unflexibel und tiefgreifend ist und ihren Beginn in Adoleszenz oder jungem Erwachsenenalter hat) und eine ausreichende Anzahl aus einem Satz von acht bis zehn Kriterien für eine spezifische PS erfüllt sind.

Für eine Borderline-Persönlichkeitsstörung (BPS) müssen fünf von neun bzw. zehn Kriterien erfüllt sein: verzweifeltes Bemühen, tatsächliches oder vermutetes Verlassenwerden zu verhindern, emotionale Instabilität, intensive und instabile zwischenmenschliche Beziehungen mit Wechsel zwischen Idealisierung und Entwertung, Identitätsstörung, Suiziddrohungen, Selbstverletzungen, Schwierigkeiten Wut zu kontrollieren, Impulsivität in potentiell selbstschädigenden Bereichen, chronische Gefühle der Leere (Falkai und Wittchen et al. 2015; WHO 1992).

Hat man mit diesen Diagnosen tatsächlich natürlich vorkommende Kategorien beschrieben und damit die Natur an ihren Gelenken getrennt („,arving nature by its joints“), wenn gleichzeitig mehr als die Hälfte der Personen, die die allgemeinen Kriterien einer PS erfüllen, in keine dieser Kategorien passen (Zimmerman et al. 2005)?

Wie unterschiedlich Personen mit der gleichen PSDiagnose sein können, ihre Heterogenität, wurde viel-

\footnotetext{
1 Die Kriterien der Persönlichkeitsstörungen im DSM-IV (1994) wurden im DSM-5 (2013) unverändert weitergeführt.
}

fach kritisch angemerkt (Ofrat et al. 2018; Widiger und Trull 2007). Für die BPS ergeben sich 256 verschiedene Symptomkonstellationen im DSM und bei fünf von neun Kriterien können zwei Patient*innen u.U. nur ein einziges Symptom überlappend präsentieren. Für die zwanghafte PS mit vier von acht Kriterien ist es sogar möglich, überhaupt keine Überlappung mit einer anderen Person zu haben, und dennoch die gleiche Diagnose zu bekommen (Samuel und Griffin 2015). Das ist nicht nur rechnerisch möglich, sondern zeigt sich auch in der klinischen Realität. In einer Stichprobe von 252 Patient*innen, die die Kriterien einer BPS erfüllten, waren 136 verschiedene Kombinationen einer BPS präsent (Johansen et al. 2004).

Dabei ist im kategorialen System die Grenze zwischen normaler und pathologischer Persönlichkeit eine willkürliche (Ofrat et al. 2018). Im „revolutionären“ DSM-III (APA 1980), das diese Art der Diagnosestellung erstmals umsetzte, wurde bei den meisten Persönlichkeitsstörungen keinerlei Begründung für die Schwelle zwischen normal und krank angegeben (Demazeux 2015). Außerdem wurde von der letztlich nicht haltbaren Position ausgegangen, dass alle Kriterien in gleichem Maße wichtig für die Diagnosestellung seien (Balsis et al. 2011). Bei der BPS begründete man im DSM-III die fünf von damals acht Kriterien zumindest als eine versuchte Maximierung der Übereinstimmung unter Kliniker*innen. Diese Form der Entscheidungsfindung wurde humoristisch als BOGSAT-Methode bezeichnet (,bunch of guys sitting around a table“; H. Pincus, zit. n. Demazeux 2015, S. 9). Damit ist die zentrale (willkürliche) Schwelle, ob jemand an einer krankheitswertigen BPS leidet, die von 4 (gesund) auf 5 (krank) erfüllte Kriterien (Samuel und Griffin 2015). Manche Autor*innen argumentieren, dass die Problematik zumindest verringert werden könnte, wenn die Anzahl der zu erfüllenden Kriterien auf mind. 7 erhöht würde (Paris 2020). Dieses strengere Vorgehen würde eine homogenere Gruppe von Personen erlauben, die eine BPS-Diagnose erhalten.

Ebenfalls sehr problematisch ist das Ergebnis von Längsschnittstudien, dass diese Kriterien in recht unterschiedlichem Maß zeitlich stabil sind und damit ein postuliertes zentrales Charakteristikum einer Persönlichkeitsstörung deutlich in Frage gestellt ist. Grilo et al. $(2004,2014)$ haben dargestellt, dass fast $30 \%$ einer nur über 24 Monate verfolgten Stichprobe von BPS-Patient*innen in diesem Zeitraum über 12 Monate und mehr nur 2 oder weniger der Kriterien erfüllten. Sie waren also über ein Jahr in diesen beiden Jahren sehr weit von einer BPS-Diagnose entfernt! So sind etwa Selbstverletzungen wesentlich weniger zeitlich stabil als die emotionale Instabilität. Umgekehrt würden jahrelange Selbstverletzungen, emotionale Instabilität und schwere Dissoziationen nicht für eine BPS-Diagnose ausreichen, auch wenn sie in andauernder schwerer Beeinträchtigung resultieren (Cooper und Balsis 2009; Samuel und Griffin 2015). 
Tyrer et al. (2019) fassen zusammen, dass die allgemeinen Kriterien einer Persönlichkeitsstörung (als Voraussetzung für die Diagnose einer spezifischen Kategorie) schlicht ignoriert worden seien (vgl. auch First et al. 2014). Wenn Kliniker*innen Diagnosen stellten, dann reduzierte sich das verwendete Spektrum auf die drei Kategorien BPS, Antisoziale PS und kombinierte PS/Nicht Näher Bezeichnete PS. Und sie betonen, dass in Studien nur bei $8 \%$ der psychiatrischen Patient*innen eine PS-Diagnose vergeben wurde, obwohl es sich empirisch gezeigt hatte, dass die realen Zahlen zwischen $40 \%$ und $90 \%$ liegen.

Diese und weitere Kritikpunkte an der kategorialen Diagnostik von Persönlichkeitsstörungen haben die Aufmerksamkeit zunehmend auf dimensionale Modelle als angemessenere Alternative gerichtet (Hopwood et al. 2018; Smith et al. 2020). Hier werden Merkmale nicht dichotom als „vorhanden“ oder „nicht vorhanden“ betrachtet. Stattdessen werden kontinuierliche Dimensionen (Traits) von leicht bis extrem identifiziert, die bei Patient*innen unterschiedlich stark ausgeprägt sind (Krueger et al. 2014). Dieser Zugang entspricht einerseits den Ergebnissen der Neurobiologie, die mit dimensionalen Modellen deutlich besser vereinbar sind als mit kategorialen (Caspi et al. 2014). Er entspricht auch den dominierenden Modellen in der Normalpsychologie der Persönlichkeit. Eine umfangreiche Integration der Persönlichkeitsstörungen mit dem führenden, empirisch sehr gut abgesicherten „Big Five“-Modell der Normalpersönlichkeit (mit den Traits „Neurotizismus“, „Extraversion“, „Offenheit“, „Verträglichkeit“ und „Gewissenhaftigkeit“) wurde erstmals bereits 1994 publiziert (Costa und Widiger 1994; Widiger und Costa 2013; Miller und Widiger 2020). Dabei wurde ein Trait definiert als eine „Variable, die einer relativ stabilen Disposition für bestimmte Verhaltensmuster zugrunde liegt“ (Markon und Jonas 2015, S. 64, dt. Übers. HM).

\section{Das dimensionale Alternative Model of Personality Disorders (AMPD) im DSM-5: die Fast-Revolution}

In der Vorbereitung des DSM-5 arbeitete die Personality \& Personality Disorder Work Group (PPDWG) unter der Leitung von Andrew Skodol klar auf die Ablöse der kategorialen Diagnostik durch ein dimensionales Modell der PS hin. Die erste konkrete Ausgestaltung (Skodol et al. 2011) sah die Streichung von fünf empirisch am schlechtesten abgesicherten Kategorien vor (paranoide, schizoide, histrionische, narzisstische und dependente PS) und beschrieb die verbleibenden PS als Prototypen auf einem Raster von Dimensionen (Krueger et al. 2014). Die Persönlichkeit von Patient*innen sollte dimensional auf 37 Facetten (z.B. emotionale Labilität, Trennungsangst, Rückzug, Feindseligkeit etc.; Skodol et al. 2011) eingeschätzt werden und diese Einschätzung sollte unabhängig von den bisherigen „Schubladen“ (antisozial, Borderline etc.) ein differen- ziertes Profil der Person liefern, mit dem die Behandlung geplant werden kann. Die Lösung war damit ein „Hybrid-Modell“ aus Prototypen auf Persönlichkeitsdimensionen, das dimensionale und kategoriale Diagnosestellung verbinden sollte und so alle, d.h. Kritiker und Vertreter des alten Modells, zufriedenstellen und den Übergang erleichtern sollte (Krueger und Markon 2014).

Das Modell löste sehr viele kritische bis negative Reaktionen aus. Sogar Widiger (2011) als langjähriger ausgewiesener Vertreter eines dimensionalen $\mathrm{Zu}$ gangs kritisierte die PPDWG, nicht das abgesicherte Big-Five-Modell als Grundlage für das DSM-5 heranzuziehen (Huprich 2015). Folge dieser heftigen Kritik war u.a. die Wiederaufnahme bzw. Beibehaltung der narzisstischen PS (ein weiteres Beispiel der BOGSATMethode?) und neuerliche Studien, die in einem System von 5 Trait-Domänen mit insgesamt 25 Facetten mündeten. Erfassungsinstrumente für diese Facetten existieren mittlerweile sowohl als Selbstbeurteilung, Fremdbeurteilung durch Angehörige sowie als klinische Einschätzung (PID-5; Personality Inventory für DSM-5; First et al. 2018, Krueger et al. 2012; Somma et al. 2019). Die PPDWG folgte nun weitgehend dem Big Five-Modell. Nicht vertreten ist die „Offenheit“, während stattdessen eine Domäne „Psychotizismus“ eingeführt wurde, um u.a. die Symptome einer Schizotypischen Persönlichkeitsstörung abbilden zu können (die im ICD-10 als Schizotypie keine PS ist; Abb. 1). Psychotizismus wurde allerdings immer wieder als mögliche Extremvariante des Traits „Offenheit“ diskutiert (vgl. Chmielewski et al. 2014).

Deutlich prominenter als im DSM-IV ist im DSM-5 die Beurteilung des Kriteriums A, die Einschätzung des Schweregrads der Beeinträchtigung der Persönlichkeit (Morey und Bender 2014). Störungen der Selbst-Funktionen (Identität und Selbststeuerung) und der zwischenmenschlichen Funktionen (Empathie und Nähe) werden differenziert und auf einem Kontinuum eingeschätzt (Tab. 1; vgl. Widiger et al. 2019 und Kommentare für eine interessante Diskussion). Erstmals wird auch die Operationalisierung der „gesunden“ Persönlichkeit inkludiert. In einer „Skala zur Erfassung des Funktionsniveaus der Persönlichkeit“ (SEFP) schätzen Kliniker*innen den Schweregrad der Beeinträchtigung von „, $0=$ keine oder geringfügige Beeinträchtigung“ bis „4=extreme Beeinträchtigung“ für die vier genannten Bereiche ein. Für die Diagnose einer Persönlichkeitsstörung müssen mindestens zwei von vier Bereichen als mindestens „2= mittelgradig“ beurteilt werden. Zur Illustration ist in Tab. 1 die „mittelgradige Beeinträchtigung“ für die vier Bereiche dargestellt (Falkai et al. 2015).

Ist die Diagnose einer Persönlichkeitsstörung vom Schweregrad der Beeinträchtigung gerechtfertigt, folgt in Kriterium B die inhaltliche Beschreibung der vorherrschenden Traits. Beschrieben sind die Domänen „Negative Affektivität“, „Verschlossenheit“, „Antagonismus“, „Enthemmtheit“ und „Psychotizismus“, die 
DSM-5

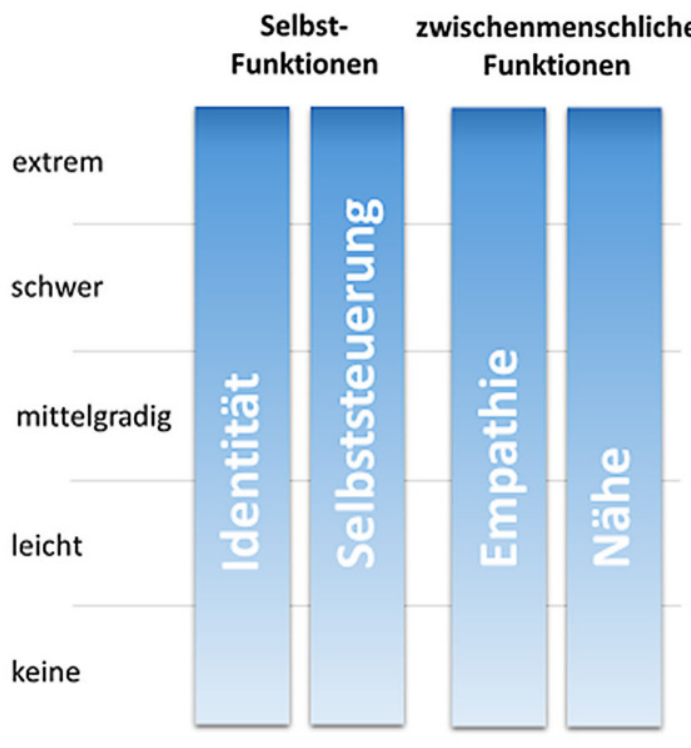

TRAIT-DOMÄNEN

\section{FACETTEN}
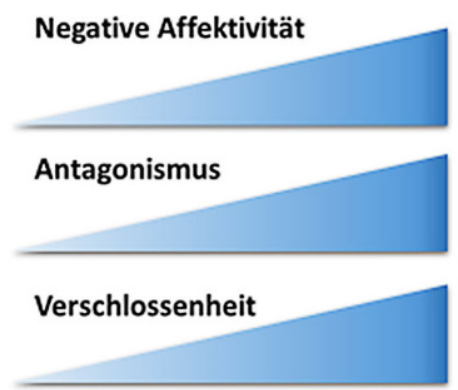

Emotionale Labilität, Ängstlichkeit, Trennungsangst, Unterwürfigkeit, Feindseligkeit, Perseveration, Affektarmut (Fehlen von )

Neigung zu Manipulation,

Unehrlichkeit, Grandiosităt, Suche nach Aufmerksamkeit, Gefühlskälte

Sozialer Rückzug, Vermeidung von Nähe, Anhedonie, Depressivität, Misstrauen

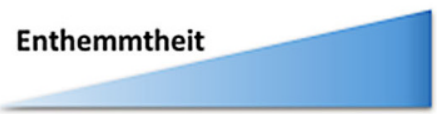

Verantwortungslosigkeit, Impulsivität, Ablenkbarkeit, Neigung zu riskantem Verhalten, Rigider Perfekionismus (Mangel an)

Ungewöhnliche Überzeugungen und innere Erlebnisse, Exzentrizität, Denkund Wahrnehmungsstörungen

\section{Beeinträchtigung}

Abb. 1 Dimensionale Einschätzung einer Persönlichkeitsstörung im DSM-5 (APA 2013; Falkai und Wittchen et al. 2015) zunächst über den Schweregrad und dann über Trait-Domänen (und Facetten). Die Einschätzung des Schweregrads erfolgt in den Bereichen „Identität“, „Selbststeuerung“, „Em- pathie" und „Nähe“ (mind. 2 Bereiche mittelgradig). Die ersten vier Trait-Domänen entsprechen dem Big Five-Modell. Die Klassifikation von 6 Prototypen (eine davon die BPS) ist hier nicht dargestellt

Tab. 1 Beispiel der „mittelgradigen Beeinträchtigung“ in der Skala zur Erfassung des Funktionsniveaus der Persönlichkeit (SEFP) im AMPD des DSM-5. (Text verkürzt; Falkai et al. 2015, S. 1066)

\begin{tabular}{l|l}
$\begin{array}{l}\text { SEFP } \\
\text { Identität }\end{array}$ & $\begin{array}{l}\text { „2= mittelgradige Beeinträchtigung“ (in zwei Bereichen notwendig für die Diagnose einer PS) } \\
\text { Vulnerabler Selbstwert, Sorge um Bewertung durch andere, Wunsch nach Anerkennung, Gefühl von Unzulänglichkeit, kompensatorisch überhöh- } \\
\text { te oder verringerte Selbsteinschätzung }\end{array}$ \\
$\begin{array}{l}\text { Selbst- } \\
\text { steuerung }\end{array}$ & $\begin{array}{l}\text { Ziele meistens nicht selbstbestimmt, sondern Mittel, Bestätigung von anderen zu erhalten, daher ev. mangelnde Stabilität von Zielen } \\
\text { Eigene Maßstäbe unangemessen hoch (z. B. gefallen wollen) oder niedrig (z. B. nicht mit vorherrschenden Werten übereinstimmend) } \\
\text { Eingeschränkte Fähigkeit, eigenes Erleben zu reflektieren }\end{array}$ \\
Empathie & $\begin{array}{l}\text { Übermäßig empfindsam hinsichtlich des Erlebens anderer, aber nur im Hinblick auf dessen Relevanz für die eigene Person } \\
\text { Übermäßig selbstbezogen, deutlich eingeschränkte Fähigkeit, das Erleben anderer zu würdigen, zu verstehen, alternative Sichtweise zu berück- } \\
\text { sichtigen } \\
\text { Ist sich der Wirkung des eigenen Verhaltens auf andere generell nicht bewusst, schätzt die Wirkung unrealistisch ein oder kümmert sich nicht } \\
\text { darum }\end{array}$ \\
Nähe & $\begin{array}{l}\text { Hat Fähigkeit und Wunsch, Beziehungen im privaten und gesellschaftlichen Umfeld aufzubauen, diese können aber größtenteils oberflächlich sein } \\
\text { Enge Beziehungen bestehen, um Bedürfnisse der Selbstregulation und des Selbstwerts zu befriedigen, verbunden mit unrealistischer Erwartung, } \\
\text { von anderen perfekt verstanden zu werden } \\
\text { Tendiert dazu, Beziehungen nicht als etwas Wechselseitiges anzusehen, sondern kooperiert vorrangig aus Eigennutz }\end{array}$
\end{tabular}

nochmals in 25 Facetten differenziert werden (um Redundanzen zu vermeiden, werden in Tab. 3 nur die Domänen des für Europa wichtigeren ICD-11 genauer dargestellt, s. unten). So kann etwa Negative Affektivität in die teilweise heterogenen Facetten „Emotionale Labilität“, „Ängstlichkeit“, „Trennungsangst“, „Unterwürfigkeit“, „Feindseligkeit“, „Perseveration“, „Depressivität“, „Misstrauen“ und (Fehlen von) „Affektarmut“ differenziert werden (Falkai et al. 2015).

Wie sieht in diesem System die Diagnose einer BPS aus? Das Hybrid-Modell (dimensional und kategori- al) im DSM-5 wird deutlich, wenn die Kriterien für das Vorliegen einer BPS als einer von sechs möglichen Prototypen beurteilt werden sollen. Diese Form der Beurteilung ist uns wieder sehr vertraut (Tab. 2):

Die BPS wird also beschrieben auf den neuen Dimensionen, die einerseits den Schweregrad der Beeinträchtigung der Persönlichkeit abbilden (Selbst und interpersonelle Beziehungen), andererseits werden jene Facetten genannt, die in den jeweiligen Trait-Domänen für die Diagnose relevant sind. Hier sind es vier von sieben Merkmalen, die erfüllt sein müssen. 
Tab. 2 Hybrid-Modell der Beurteilung einer BorderlineStörung im alternativen Modell der Persönlichkeitsstörungen des DSM-5. (Text deutlich verkürzt; Falkai et al. 2015, S. 1053,1054$)$

A. Mittelgradige oder stärkere Beeinträchtigung im Funktionsniveau der Persönlichkeit Schwierigkeiten in mind 2 der folgenden Bereiche:

1. Identität: verarmtes, instabiles Selbstbild, exzessive Selbstkritik, chronische Gefühle innerer Leere, bei Belastung Dissoziation

2. Selbststeuerung: Instabilität in Zielsetzungen, Vorlieben, Wertvorstellungen und beruflichen Plänen

3. Empathie: Eingeschränkte Fähigkeit, die Gefühle und Bedürfnisse anderer Personen zu erkennen, verbunden mit zwischenmenschlicher Überempfindlichkeit

4. Nähe: Intensive, aber instabile und konfliktreiche enge zwischenmenschliche Beziehungen, die durch Misstrauen und ängstliche Beschäftigung mit Verlassenwerden gekennzeichnet sind

B. Mindestens 4 der folgenden 7 Persönlichkeitsmerkmale, mindestens eines ist davon (5) Impulsivität, (6) Neigung zu riskantem Verhalten oder

(7) Feindseligkeit

1. Emotionale Labilität (Negative Affektivität)

2. Ängstlichkeit (Negative Affektivität)

3. Trennungsangst (Negative Affektivität)

4. Depressivität (Negative Affektivität)

5. Impulsivität (Enthemmtheit)

6. Neigung zu riskantem Verhalten (Enthemmtheit)

7. Feindseligkeit (Antagonismus)

Sechs der bisherigen 10 Typen wurden in das Hybrid-Modell aufgenommen (antisozial, vermeidendselbstunsicher, Borderline, narzisstisch, zwanghaft, schizotyp). Sollte keiner dieser Prototypen angemessen sein, ist die Diagnose einer Persönlichkeitsstörung, Merkmalsspezifiziert (PS-MS) möglich. Sie entspricht am ehesten einem rein dimensionalen Modell: (1) allgemeine Einschätzung der Schwere der Persönlichkeitsstörung (Kriterium A; mind. zwei von vier Bereichen mind. mittelgradig) und (2) spezifische Einschätzung der fünf Traits (Kriterium B; Negativität, Verschlossenheit, Antagonismus, Enthemmtheit und Psychotizismus; Falkai et al. 2015, S. 1058).

Der Schweregrad (Kriterium A) und Traits (Kriterium B) sollten damit am besten unabhängig voneinander sein, was sich empirisch wahrscheinlich nicht halten lässt (Bastiaansen et al. 2016; Berghuis et al. 2014; Zimmermann et al. 2015). Im Gegenteil bildet die Gesamtanzahl der erfüllten PS-Kriterien (A und B zusammen im Gegensatz zu A allein) am besten die gegenwärtigen und zukünftigen Belastungen in Arbeit, Freizeit und Beziehungen ab (Hopwood et al. 2011). Es schält sich also neben den spezifischen Faktoren eine Art allgemeiner Faktor heraus, auf dem alle Kriterien (A und B) laden. Interessanterweise scheinen die Kriterien der BPS auf diesem allgemeinen Faktor eher zu laden als auf spezifischen Traits (Sharp et al. 2015; Wright et al. 2016). Manche Autor*innen kritisieren daher, dass etwa die narzisstische oder die antisoziale PS sehr gut als ich-syntone Extremvarianten von Traits betrachtet werden können, was allerdings für die BPS nicht zuzutreffen scheint. Eine Reihe von BPS-Symptomen können nicht gut auf diesem System abgebildet werden. Diese Symptome werden zudem als stark ichdyston erlebt werden (z. B. chronische Suizidalität, Depersonalisation, Pseudohalluzinationen; Paris 2020).
Konkrete Anwendungsbeispiele des AMPD in Assessment und Behandlungsplanung finden sich z.B. bei Bach et al. (2015), Pincus et al. (2016) oder Waugh et al. (2017).

Zum Modell entwickelte Selbstbeurteilungsinstrumente für die klinische Erfassung des Schweregrads (Levels of Personality Functioning Scale, Morey 2017; Kurzversion LPFS-BF, Bach und Hutsebaut 2018) und der Trait-Domänen (Personality Inventory für DSM-5 - PID-5 und Kurzversionen; Krueger et al. 2012; Somma et al. 2019) werden aktuell intensiv untersucht, sind aber noch nicht normiert. Diese PID-Versionen sind von der American Psychiatric Association für Forschungszwecke und klinische Evaluation kostenlos und auf Deutsch im Netz zur Verfügung gestellt. Zusammenfassende Darstellungen von Messinstrumenten zu beiden Diagnosesystemen finden sich bei Hengartner et al. (2018), Zimmermann et al. (2019) und Evans et al. (2020).

Hopwood (2018) hat außerdem erstmals versucht, den Einsatz des AMPD über einzelne Fallvignetten hinaus für die Auswahl von therapeutischen Techniken $\mathrm{zu}$ illustrieren. Ein wesentlicher Kritikpunkt an kategorialen Modellen war ja das Fehlen von evidenzbasierten Behandlungsmaßnahmen für die meisten Persönlichkeitsstörungen mit Ausnahme der BPS (Cristea et al. 2017). Dimensionale Modelle sollten hier eine wesentlich sinnvollere (und schließlich evidenzbasierte) Basis für die Wahl von Behandlungsmethoden liefern. Hopwood (2018) hat ein noch sehr vorläufiges Modell präsentiert, das auf den ersten Blick noch ungewohnt und etwas kantig wirken mag. Aber wir werden uns vielleicht daran gewöhnen (müssen), in Zukunft Manuale mit Titeln wie „Therapie der Negativen Affektivität“, „Therapie der Verschlossenheit“ oder „Therapie der Enthemmung“ zu finden. Auch wenn es eine sehr spannende Diskussion bleibt, welche Aspekte eines (zeitlich stabilen) Traits einer Veränderung durch Therapie zugänglich sind (Ringwald et al. 2020), zeigen Reviews, dass Interventionen Wirkung auf Traits zeigen (Roberts et al. 2017), und erste Guidelines bzw. Vorschläge für den klinischen Umgang mit verschiedenen Traits wurden bereits formuliert (Bach und Presnall-Shvorin 2020).

Was das Board of Trustees der American Psychiatric Association für das DSM-5 vollinhaltlich abgelehnt und in den Teil III („Emerging models“) verbannt hat, während im Teil II nach wie vor die alte kategoriale Diagnostik zu finden ist (Krueger und Markon 2014), wurde in der ICD-11 vollinhaltlich umgesetzt und ist seit Mai 2019 von der WHO abgesegnet. Wenn die ICD-11 ab dem Jahr 2022 das gültige Diagnosesystem darstellen wird, verändert sich die Diagnosestellung der Persönlichkeitsstörungen weltweit grundlegend. Damit wird die offizielle Diagnostik der PS in den USA letztlich fundamental inkonsistent mit der Diagnosestellung im Rest der Welt (Bagby und Widiger 2020). 
Paradigmenwechsel in der ICD-11: Dimensionale Klassifikation der Persönlichkeitsstörungen

Die Arbeiten an der Diagnostik der Persönlichkeitsstörungen begannen im Jahr 2010 noch vor der Publikation des DSM-5 (Tyrer et al. 2019). Dabei lehnte die Arbeitsgruppe von Beginn an ein Hybrid-Modell wie im DSM-5 ab und legte stattdessen eine einzelne Dimension für den Schweregrad der PS von „normal“ bis „schwer“ fest. Die Beeinträchtigung wurde über das Maß der interpersonellen Dysfunktion, die Folgen für soziale und berufliche Rollen, kognitive und emotionale Erfahrungen und Risiko, sich selbst oder anderen zu schaden, erfasst. Hauptgrund für die herausragende Betonung einer einzelnen Dimension für den Schweregrad einer PS war die bisherige hohe Komorbidität von PS-Diagnosen, die beliebig angesetzte Schwelle zwischen "gesund“ und „krank“ und die schlechte Abdeckung der Symptome von Patient*innen in den 10 Kategorien (Ofrat et al. 2018; s. oben). Außerdem zeigte sich immer wieder, dass mit zunehmendem Schweregrad auch die Anzahl der PS-Diagnosen zunahm. Kliniker*innen wählten daher der Einfachheit halber (ev. zusätzlich) die Nicht Näher Bezeichnete PS in komplexen Fällen (Tyrer et al. 2019).

Bach und First (2018) beschreiben die Anwendung des neuen Modells in der klinischen Praxis. Hier ist in einem ersten Schritt zunächst zu überprüfen, ob die allgemeinen Anforderungen an die Diagnose einer Persönlichkeitsstörung erfüllt sind. Gefordert ist eine überdauernde Störung, charakterisiert durch Probleme im Funktionsniveau von Aspekten des Selbst (z. B. Identität, Selbstwert, Akkuratheit der Selbstwahrnehmung, Selbststeuerung) und/oder interpersonelle
Dysfunktion (z. B. Fähigkeit, enge und wechselseitig befriedigende Beziehungen herzustellen, die Perspektive des anderen zu verstehen und Konflikte handzuhaben).

Diese Störung muss nicht mehr in der Jugend oder im jungen Erwachsenalter beginnen, sie muss nur länger als 2 Jahre andauern. Damit sind erstmals auch PS möglich, die mit dem 50. Lebensjahr ihren Anfang nehmen! Wenn vor dem 25. Lebensjahr keine Auffälligkeiten erkennbar sind, ist ein Qualifier „später Beginn" geplant (Tyrer et al. 2015).

Die Störung manifestiert sich in kognitiven Mustern, emotionalem Erleben und Ausdruck und ist über verschiedene persönliche und soziale Situationen erkennbar. Sie ist nicht entwicklungsgemäß oder durch soziale oder kulturelle Faktoren (inkl. politische Konflikte) erklärbar. Sie ist nicht Ausdruck einer Erkrankung, einer Medikamentenwirkung oder eines Entzugs und verursacht erhebliche persönliche, familiäre, soziale und berufliche Belastung.

In einem zweiten zentralen Schritt wird der Schweregrad einer PS eingeschätzt. Praktiker*innen haben hier auch keine andere Wahl, zu einer Diagnose zu kommen, weil die 10 Kategorien der ICD-10 nicht länger vergeben werden können. Damit wird das Problem der Komorbidität erheblich reduziert und es findet nur mehr die Einschätzung von „mild“ (6D10.0) über „moderat“ (6D10.1) bis „schwer“ (6D10.2) statt. Zusätzlich kann auch „Schweregrad unspezifiziert“ (6D10.Z) und eine subklinische Kategorie „Schwierigkeit" (difficulty) vergeben werden (Abb. 2). Hier gibt es also Auffälligkeiten, die ansatzweise Belastung verursachen, aber nicht so deutlich sind, dass sie die Diagnose einer Störung rechtfertigen (Tyrer et al. 2015). Das dazu entwickelte „Standardized Assess-
Abb. 2 Dimensionale Einschätzung der Persönlichkeitsstörung in der ICD11 (Tyrer et al. 2019) zunächst über Schweregrad und dann die Trait-Domänen. Auf eine Beschreibung von Facetten wurde verzichtet, um das Modell möglichst einfach zu halten. Die ersten vier Domänen entsprechen dem Big FiveModell. Die Domänen „Enthemmtheit“ und „Zwangshaftigkeit" sind möglicherweise die entgegengesetzten Pole einer einzigen bipolaren Domäne
ICD-11 SCHWEREGRAD

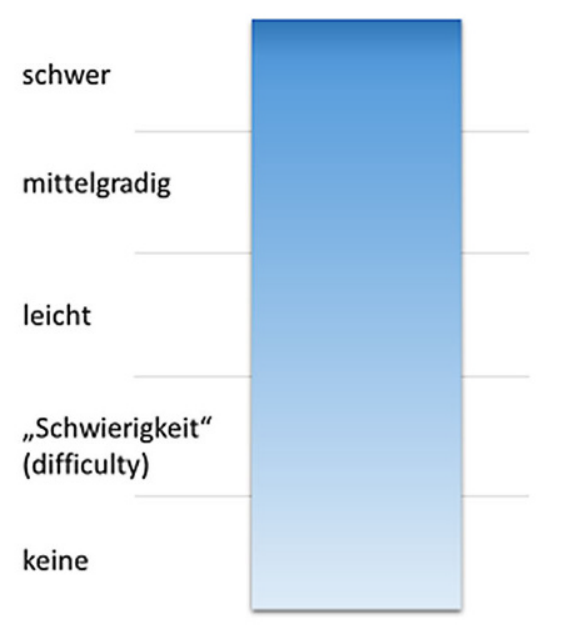

Beeinträchtigung
TRAIT-DOMÄNEN

FACETTEN

\section{Negative Affektivität}
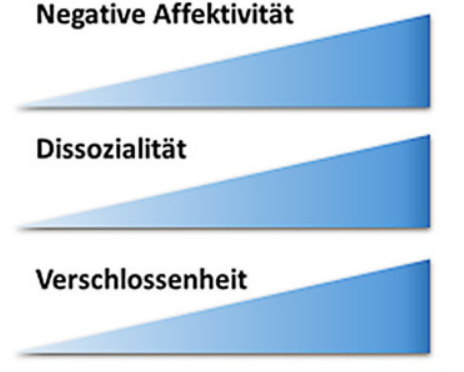

Enthemmtheit
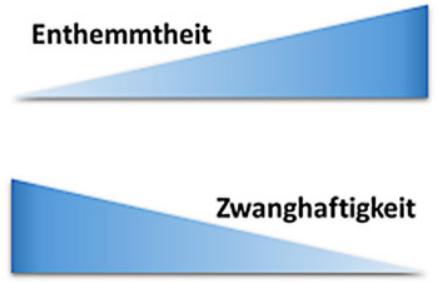

$--$

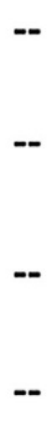

$-$ 
Tab. 3 Trait-Domänen der Persönlichkeitsstörungen im ICD-11 (Tyrer et al. 2015, S. 723; dt. Übers. HM)

\begin{tabular}{|c|c|}
\hline $\begin{array}{l}\text { Negative Affekti- } \\
\text { vität }\end{array}$ & $\begin{array}{l}\text { Hpts. charakterisiert durch die Tendenz, eine Bandbreite von belastenden Emotionen zu zeigen/erleben (manifest) wie Angst, Ärger, Selbst- } \\
\text { verachtung, Gereiztheit, Verletzlichkeit, Depression und andere negative emotionale Zustände, häufig als Reaktion auf sogar relativ kleine } \\
\text { tatsächliche oder wahrgenommene Stressoren }\end{array}$ \\
\hline Dissozialität & $\begin{array}{l}\text { Kern dieser Trait-Domäne ist die Nicht-Beachtung von sozialen Normen und Konventionen und der Rechte und Gefühle von anderen. Traits } \\
\text { in dieser Domäne inkludieren Gefühlskälte, Fehlen von Empathie, Feindseligkeit und Aggression, Rücksichtslosigkeit und die Unfähigkeit } \\
\text { oder Unwilligkeit, prosoziales Verhalten aufrechtzuerhalten, oft manifestiert als ein übermäßig positives Selbstbild, Ansprüchlichkeit und } \\
\text { einer Tendenz, andere zu manipulieren und auszunützen }\end{array}$ \\
\hline Verschlossenheit & $\begin{array}{l}\text { Kern dieser Domäne ist emotionale und zwischenmenschliche Distanz, manifestiert in deutlichem sozialem Rückzug und/oder Indifferenz } \\
\text { anderen gegenüber, Isolation mit sehr wenigen oder keinen Bezugspersonen, inkl. Vermeidung nicht nur von intimen Beziehungen, sondern } \\
\text { auch Freundschaften. Traits in dieser Domäne schließen Distanz oder Kälte in der Beziehung zu anderen Personen, Reserviertheit, Passivität } \\
\text { und Fehlen von Selbstsicherheit und reduziertes Erleben und Ausdrücken von Emotionen, besonders von positiven Emotionen mit ein, in } \\
\text { einem Ausmaß, dass die Fähigkeit, Genuss zu empfinden, verringert ist }\end{array}$ \\
\hline Enthemmheit & $\begin{array}{l}\text { Charakterisiert durch eine persistierende Tendenz, auf unmittelbare internale oder Umwelt-Stimuli impulsiv zu reagieren, ohne längerfristige } \\
\text { Konsequenzen zu berücksichtigen. Traits in dieser Domäne schließen Verantwortungslosigkeit, Impulsivität ohne Beachtung von Risiko oder } \\
\text { Konsequenzen, Ablenkbarkeit und Leichtsinnigkeit mit ein }\end{array}$ \\
\hline $\begin{array}{l}\text { Zwanghaftigkeit } \\
\text { (Anankastia) }\end{array}$ & $\begin{array}{l}\text { Kern dieser Trait-Domäne ist ein enger Fokus auf Kontrolle und die Regulation des eigenen und des Verhaltens Anderer, um sicherzustellen, } \\
\text { dass Dinge nach der besonderen Vorstellung bzw. dem Ideal der Person ablaufen. Traits in dieser Domäne inkludieren Perfektionismus, } \\
\text { Perseveration, restriktive Emotionalität und Verhalten, Starrköpfigkeit, Überlegtheit, Ordnungsliebe und Beschäftigt-sein mit dem Befolgen } \\
\text { von Regeln und dem Nachkommen von Normen }\end{array}$ \\
\hline
\end{tabular}

ment of Personality Disorder“ (SASPD; Olajide et al. 2018) ist ein sehr kurzes Selbstbeurteilungsinstrument mit nur 9 Items zur Erfassung des Schweregrades (vgl. auch Bach und Anderson 2020 für einen Vergleich der Instrumente für das DSM-5 und der ICD-11).

In einem dritten Schritt kann der Persönlichkeitsstil einer Person beschrieben werden. Die Traits in der ICD-11 entsprechen weitgehend den DSM-5-Domänen. Ursprünglich wurden die vier Traits „Negative Affektivität“ (6D11.0), „Verschlossenheit“ (6D11.1), „Dissozialität“ (6D11.2) und „Zwanghaftigkeit (Anankastia)“ (6D11.4) vorgeschlagen. Die fünfte Dimension „Enthemmtheit“ (6D11.3) wurde erst später hinzugefügt (Tyrer et al. 2019; Tab. 3). Die Unabhängigkeit der beiden letzten Traits wird allerdings in Zweifel gezogen. Empirische Ergebnisse weisen darauf hin, dass Zwanghaftigkeit und Enthemmtheit zwei entgegengesetzte Pole der gleichen Dimension sein könnten (Bach et al. 2020; Oltmanns und Widiger 2020).

Diese Beschreibung des Stils der Persönlichkeit ist für das Stellen einer Diagnose nicht notwendig (dies im Unterschied zum DSM-5; Tab. 2)! Es ist zwar wahrscheinlich, dass mit zunehmendem Schweregrad der PS auch die Anzahl der auffälligen Trait-Domänen ansteigt (Tyrer et al. 2019), die zum spezifischen Ausdruck der Dysfunktion beitragen. Aber es ist ohne weiteres möglich und zulässig, dass bei einer schweren PS nur eine oder keine Domäne markant im Vordergrund steht. Die Domänen werden als kontinuierliche Dimensionen, nicht als Kategorien verstanden. Dennoch muss für den Zweck der Kodierung die Entscheidung „vorhanden“ vs. „nicht vorhanden“ getroffen werden (Bach und First 2018). Die Autoren beschreiben in ihrer Illustration des Vorgehens fünf verschiedene Patient*innen, von denen eine dargestellte Person unter einer erheblichen Beeinträchtigung durch eine Borderline-Symptomatik leidet. Die Beeinträchtigung wurde bei ihr als „schwer“ eingeschätzt. Von den Trait-Domänen wurden (1) Negative Affektivität, (2) Dissozialität und (3) Enthemmung als „vor- handen“ gewertet, d.h. also eine schwere Persönlichkeitsstörung geprägt durch viele negative Emotionen, wie Scham, Ärger und Misstrauen, Enthemmung als impulsive und schädliche Reaktionen auf unmittelbare Stimuli und Dissozialität als misstrauische Aggression und Tendenz andere zu manipulieren. So könnte also in Zukunft die dimensionale Beschreibung einer BPS aussehen.

Während im AMPD neben den Domänen ein deutlich differenzierteres Level von 25 Facetten beschrieben wurde (Krueger und Markon 2014), hat die ICD11 Work Group bewusst auf Facetten verzichtet, um die klinische Anwendung möglichst einfach zu halten (Tyrer et al. 2011; Abb. 2). Allerdings haben Oltmanns und Widiger (2020) z.B. für die Domäne „Negative Affektivität“ klar argumentiert, dass viele der bisherigen PS-Kategorien negative Emotionen beinhalten (narzisstische, antisoziale, selbstunsichere, zwanghafte, abhängige, Borderline-PS) und eine Unterscheidung der Affekte (externalisierend: Ärger, Misstrauen vs. Internalisierend: Angst, Depression etc.; vgl. auch Smith et al. 2020) wesentlich zur klinischen Nützlichkeit des Systems beitragen würde. Daher haben sie nur 2 Jahre nach der Publikation ihres „Personality Inventory for ICD-11“ (PiCD; Oltmanns und Widiger 2018) mit dem „Five-Factor Personality Inventory for ICD11 “ (FFiCD) eine Erweiterung veröffentlicht, die die Trait-Domänen auf Facetten-Niveau erfasst (Gesamt 20 Facetten, 7 für Negative Affektivität, je 3 für Dissozialität, Verschlossenheit und Zwanghaftigkeit und 4 für Enthemmung), die überwiegend auch in einer Faktorenanalyse bestätigt werden konnten (Oltmanns und Widiger 2020). Diese Erweiterung ist für eine Diagnosestellung nach ICD-11 nicht relevant, sollte sich aber als klinisch nützlich und behandlungsrelevant erweisen. Tyrer et al. (2019) betonen jedenfalls, dass auch das vorgeschlagene System ohne Facetten ausreichend vielschichtig sei, wenn bei drei Schweregraden (plus der „Schwierigkeit“ als subklinisches Phänomen) und fünf Trait-Dimensionen fast 500 unter- 
Tab. 4 Beschreibung des Borderline-Musters in der ICD11 (Tyrer et al. 2019; Bach und First 2018, dt. Übers. HM)

Tiefgreifendes Muster charakterisiert von Instabilität in zwischenmenschlichen Beziehungen, Selbstbild, Affekten und Impulsivität (sichtbar durch das Vorhandensein von vielen [sic!] der folgenden Punkte):

Heftige Versuche reales oder vorgestelltes Verlassenwerden zu vermeiden Muster instabiler und intensiver zwischenmenschlicher Beziehungen Identitätsstörung als deutliches und persistierendes instabiles Selbstbild Tendenz, bei starken negativen Emotionen impulsiv zu handeln, führt zu potenziell selbstschädigendem Verhalten

Wiederkehrende Episoden von Selbstschädigung

Emotionale Instabilität aufgrund von deutlicher Reaktivität der Stimmung

Chronische Gefühle der Leere

Unangemessener intensiver Ärger oder Schwierigkeiten Ärger zu kontrollieren

Vorübergehende dissoziative Zustände oder psychoseähnliches Erleben in Situationen mit hoher emotionaler Anspannung

Andere Manifestationen des Borderline-Musters, die nicht alle zum gegebenen Zeitpunkt präsent sein müssen:

Ein Selbstbild als unzulänglich, schlecht, schuldig, abstoßend und verachtenswert

Erleben des Selbst als grundlegend anders und isoliert von anderen Menschen, schmerzliches Gefühl der Entfremdung und tiefe Einsamkeit Hypersensitivität für Zurückweisung, Probleme, angemessen Vertrauen in zwischenmenschlichen Beziehungen aufzubauen bzw. aufrecht zu erhalten häufige Fehlinterpretation von sozialen Signalen

schiedliche diagnostische Ergebnisse erreicht werden können und auch die BPS im System sehr gut abgebildet werden könne.

Die Akzeptanz dieses Vorgehens im ICD-11 (wie auch im AMPD) wird wesentlich davon abhängen, ob und in welcher Form in Zukunft Behandlungsentscheidungen durch die Einschätzung des Schweregrads bzw. der Domänen erleichtert werden (vgl. Bach und Presnall-Shvorin 2020). Die postulierten DSM-5 und ICD-11 Trait-Domänen scheinen jedenfalls immer wieder empirisch erhärtet werden $\mathrm{zu}$ können und wurden auch in verschiedenen Kulturen repliziert (Bach et al. 2017; Lotfi et al. 2018; Pires et al. 2017). Es gibt aber auch viele kritische Stimmen wie Paris (2020), der zwar grundsätzlich die Entwicklung begrüßt, jedoch Jahrzehnte von Forschung in Gefahr oder sogar verloren sieht. Dies betrifft v.a. die BPS, für die innerhalb der spezifischen PS mit Abstand die meiste Forschungsliteratur entstanden sei.

Und deshalb haben gerade hier (und nur hier) bei der BPS Kritiker*innen des Modells (Herpertz et al. 2017) erreicht, dass sie als „Borderline-Muster“ (borderline pattern) im ICD-11 erhalten bleibt. Die Kritiker*innen wendeten ein, dass ein Diagnosesystem über die wissenschaftlichen Kriterien hinaus klinische Nützlichkeit haben sollte und so hilfreiche Informationen zur Wahl von Behandlungen, Prognose und Outcomes liefern sollte. Sie sollte Patient*innen helfen, informierte Zustimmung geben zu können bzw. entscheiden zu können, welche Behandlungen ihnen am ehesten hilfreich erscheinen. Es sollte Ressourcen im Gesundheitssystem sinnvoll einsetzen helfen und sollte getragen sein von robusten wissenschaftlichen Ergebnissen. Diese Aspekte sahen die Autor*innen im Vorschlag für die ICD-11 nicht wirklich erfüllt (Herpertz et al. 2017). Dabei unterstützen auch diese Kriti- ker*innen grundsätzlich einen dimensionalen Ansatz, fordern aber ein Hybrid-Modell wie im DSM-5, um einen schonenderen Übergang zu gewährleisten.

Tyrer et al. (2019) beschreiben die Diskussion und deren Lösung durch Aufnahme des Borderline-Qualifiers als einen politischen Kompromiss, um die Akzeptanz des neuen Systems möglichst zu stärken. Der Verlust der BPS erschiene den Kritiker*innen zu radikal, weil viele Forschungsgelder und Projekte auf dieser Basis entstanden seien und wichtige Forschung dadurch initiiert worden sei. Zwar gäbe es nur wenig Forschung in der Klassifikation der BPS, aber Forschung in ihre Behandlung sei in großem Maß entstanden und habe Optimismus aufgebaut. Im Wesentlichen ist das Borderline-Muster eine Neuformulierung und Update der Kriterien in der ICD-10 (Tab. 4).

Tyrer selbst sei den Diskussionen über diese Lösung bewusst ferngeblieben, nachdem er sich mehrfach klar dagegen ausgesprochen habe. Jedoch sehe die ICD-11 Working Group die exzellente Gelegenheit, die beiden Herangehensweisen der Diagnosestellung miteinander zu vergleichen. Ihre Hoffnung ist es, dass die Möglichkeit, die BPS auf den Dimensionen gut darstellen zu können, die Bedeutung des Borderline-Musters für die Diagnose zurückgehen lässt (Tyrer et al. 2019).

Der Wechsel auf ein dimensionales Modell in den Persönlichkeitsstörungen wurde wiederholt und wohl zu Recht als Paradigmenwechsel bezeichnet. Das völlige Aufgeben der sehr vertrauten Kategorien einer narzisstischen, einer ängstlich-vermeidenden oder einer dependenten PS ist ein überraschend weitreichender und gleichzeitig gut argumentierter Schritt, den man in dieser Radikalität nicht ohne weiteres für möglich gehalten hätte. Es ist ein fundamentaler Wechsel hin zu einem empirisch unterstützten diagnostischen System, der eine massive Veränderung bestehender Forschungstraditionen bedeutet, aber gleichzeitig einen generellen Übergang zu dimensionalen Modellen in der Beschreibung von Psychopathologie allgemein einläuten könnte (Kotov et al. 2017; Smith et al. 2020). Unter dem Begriff „Hierarchical Taxonomy Of Psychopathology“ (HiTOP; http:// medicine.stonybrookmedicine.edu/HiTOP) bemüht sich eine Gruppe von renommierten Forscher*innen um diesen radikalen Übergang und bereitet ein allgemeines dimensionales Klassifikationssystem für alle psychischen Symptome vor, das eine Vielzahl von empirischen Befunden $\mathrm{zu}$ integrieren versucht. Die Forscher*innen haben die Hoffnung, dadurch neue nosologische Forschung zu stimulieren und zu leiten (Kotov et al. 2017). Die dimensionale Diagnostik der Persönlichkeitsstörungen wird hier zur „Vorreiterin in der Post-DSM-5-Ära“ (Krueger 2013). Natürlich ist abzuwarten, wie ihre endgültige Ausgestaltung im ICD-11 aussehen wird. Aber es ist jetzt schon klar, dass die wissenschaftliche Psychologie im Wettstreit mit der klassischen psychiatrischen Nosologie markante Akzente gesetzt hat. Nun bleibt abzuwarten 
und $\mathrm{zu}$ hoffen, dass sich dies - neben Innovationen in der Forschung - auch in einer weiteren Verbesserung der psychotherapeutischen Behandlung und Versorgung unserer (B)PS-Patient*innen niederschlagen wird. Davon wird wesentlich abhängen, wie sehr diese spannende Entwicklung an Fahrt aufnimmt.

Funding Open access funding provided by University of Innsbruck and Medical University of Innsbruck.

Interessenkonflikt $\mathrm{H}$. Mitmansgruber gibt an, dass kein Interessenkonflikt besteht.

Open Access Dieser Artikel wird unter der Creative Commons Namensnennung 4.0 International Lizenz veröffentlicht, welche die Nutzung, Vervielfältigung, Bearbeitung, Verbreitung und Wiedergabe in jeglichem Medium und Format erlaubt, sofern Sie den/die ursprünglichen Autor(en) und die Quelle ordnungsgemäß nennen, einen Link zur Creative Commons Lizenz beifügen und angeben, ob Änderungen vorgenommen wurden.

Die in diesem Artikel enthaltenen Bilder und sonstiges Drittmaterial unterliegen ebenfalls der genannten Creative Commons Lizenz, sofern sich aus der Abbildungslegende nichts anderes ergibt. Sofern das betreffende Material nicht unter der genannten Creative Commons Lizenz steht und die betreffende Handlung nicht nach gesetzlichen Vorschriften erlaubt ist, ist für die oben aufgeführten Weiterverwendungen des Materials die Einwilligung des jeweiligen Rechteinhabers einzuholen.

Weitere Details zur Lizenz entnehmen Sie bitte der Lizenzinformation auf http://creativecommons.org/licenses/by/4. 0/deed.de.

\section{Literatur}

American Psychiatric Association (1980). Diagnostic and statistical manual of mental disorders (3.Aufl.). Washington: APA.

American Psychiatric Association (1994). Diagnostic and statistical manual of mental disorders (4.Aufl.). Washington: APA.

American Psychiatric Association (2013). Diagnostic and statistical manual of mental disorders (5.Aufl.). Washington: APA.

Bach, B., \&Anderson, J. L. (2020). Patient-reported ICD-11 personality disorder severity and DSM-5 level of personality functioning. Journal of Personality Disorders, 34,231-249. https://doi.org/10.1521/pedi_2018_32_393.

Bach, B., \& First, M.B. (2018). Application of the ICD-11 classification of personality disorders. BMC Psychiatry, 18,351.https://doi.org/10.1186/s12888-018-1908-3.

Bach, B., \& Hutsebaut, J. (2018). Level of personality functioning scale-brief form 2.0: utility in capturing personality problems in psychiatric outpatients and incarcerated addicts. Journal of Personality Assessment, 100, 660-670. https://doi.org/10.1080/00223891.

Bach, B., \& Presnall-Shvorin, J. (2020). Using DSM-5 and ICD11 personality traits in clinical treatment. In C. W. Lejuez \& K. Gratz (Hrsg.), The Cambridge handbook of personality disorders (S. 450-467). Cambridge: Cambridge University Press.

Bach, B., Sellbom, M., Kongerslev, M., et al. (2017). Deriving ICD-11 personality disorder domains from DSM-5 traits: Initial attempt to harmonize two diagnostic systems. Acta
Psychiatrica Scandinavica, 136, 108-117.https://doi.org/ 10.1111/acps.12748.

Bach, B., Christensen, S., Kongerslev, M. T., Sellbom, M., \& Simonsen, E. (2020). Structure of clinician-reported ICD-11 personality disorder trait qualifiers. Psychological Assessment, 32, 50-59. https://doi.org/10.1037/pas0000747.

Bach, B., Markon, K., Simonsen, E., \& Krueger, R. F. (2015). Clinical utility of the DSM-5 alternative model of personality disorders: six cases from practice. Journal of Psychiatric Practice, 21,3-25.

Bagby, R. M., \& Widiger, T.A. (2020). Assessment of the ICD11 dimensional trait model: an introduction to the special section. Psychological Assessment, 32, 1-7. https://doi. $\operatorname{org} / 10.1037 /$ pas0000785.

Balsis, S., Lowmaster, S., Cooper, L. D., \& Benge, J.F. (2011). Personality disorder diagnostic tresholds correspond to different levels of latent pathology. Journal of Personality Disorders, 25, 115-127. https://doi.org/10.1521/pedi. 2011.25.1.115.

Bastiaansen, L., Hopwood, C. J., Van den Broeck, J., Rossi, G., Schotte, C., \& De Fruyt, F. (2016). The twofold diagnosis of personality disorder: How do personality dysfunction and pathological traits increment each other at successive levels of the trait hierarchy? Personality Disorders: Theory, Research, and Treatment, 73, 280-292. https://doi.org/10. $1037 /$ per0000149.

Berghuis, H., Kamphuis, J.H., \& Verheul, R. (2014). Specific personality traits and general personality dysfunction as predictors of the presence and severity of personality disorders in a clinical sample. Journal of Personality Assessment, 96,410-416.https://doi.org/10.1080/00223891. 2013.834825.

Caspi, A., Houts, R. M., Belsky, D. W., et al. (2014). The p factor: One general psychopathology factor in the structure of psychiatric disorders? Clinical Psychological Science, 2, 119-137.https://doi.org/10.1177/2167702613497473.

Chmielewski, M., Bagby, R. B., Markon, K., Ring, A. J., \& Ryder, A. G. (2014). Openness to experience, intellect, schizotypal personality disorder, and psychoticism: resolving the controversy. Journal of Personality Disorders, 28, 483-499. https://doi.org/10.1521/pedi_2014_28_128.

Cooper, L.D., \& Balsis, S. (2009). When less is more: how fewer diagnostic criteria can indicate greater severity. Psychological Assessment, 21, 285-293. https://doi.org/ $10.1037 / \mathrm{a} 0016698$.

Costa, P. T. Jr., \& Widiger, T. A. (1994). Personality disorders and the five-factor model of personality. Washington: American Psychological Association.

Cristea, I.A., Gentilo, C., Cotet, C.D., Palomba, D., Barbui, C., \& Cuijpers, P. (2017). Efficacy of psychotherapies for borderline personality disorder: a systematic review and meta-analysis. JAMA Psychiatry, 74, 319-328. https:// doi. org/10.1001/jamapsychiatry.2017.1403.

Demazeux, S. (2015). The ideal of scientific progress in the DSM. In S. Demazeux \& P. Singy (Hrsg.), The DSM-5 in perspective: philosophical reflections on the psychiatric babel (S.3-24). Dordrecht: Springer.

Evans, C. M., Williams, T. F., \& Simms, L. J. (2020). Methods and current issues in dimensional assessment of personality pathology. In C. W. Lejuez \& K. Gratz (Hrsg.), The Cambridge handbook of personality disorders (S. 329-346). Cambridge: Cambridge University Press.

Falkai, P., \& Wittchen, H.-U., et al. (2015). Diagnostisches und Statistisches Manual psychischer Störungen DSM-5. Göttingen: Hogrefe.

First, M.B., Bhat, V., Adler, D., Dixon, L., \& Goldman, B. (2014). How do clinicians actually use the Diagnostic and 
Statistical Manual of Mental Disorders in clinical practice and why we need to know more. Journal of Nervous and Mental Disease, 202, 841-844. https://doi.org/10.1097/ NMD.0000000000000210.

First, M. B., Skodol, A., Bender, D. S., \& Oldham, J. (2018). Structure clinical interview for the DSM-5 alternative model for personality disorders (SCID-AMPD). Arlington: American Psychiatric Association.

Grilo, C. M., Shea, M. T., Sanislow, C. A., et al. (2004). Two-year stability and change in schizotypal, borderline, avoidant, and obsessive-compulsive personality disorders. Journal of Consulting and Clinical Psychology, 72, 767-775. https://doi.org/10.1037/0022-006X.72.5.767.

Grilo, C. M., McGlashan, T. H., \& Skodol, A.E. (2014). Course and outcome. In J. M. Oldham, A. E. Skodol \& D. S. Bender (Hrsg.), The American textbook of personality disorders (2. Aufl. S. 165-186). Washington: American Psychiatric Publishing.

Hengartner, M. P., Zimmermann, J., \&Wright, A. C. (2018). Personality pathology. In V. Zeigler-Hill \& T. K. Shackelford (Hrsg.), The SAGE Handbook of personality and individual differences (Bd. 3, S. 3-35). London: SAGE.

Herpertz, S. C., Huprich, S. K., Bohus, M., etal. (2017). The challenge of transforming the diagnostic system of personality disorders. Journal of Personality Disorders, 31, 577-589. https://doi.org/10.1521/pedi_2017_31_338.

Hopwood, C. J. (2018). A framework for treating DSM-5 alternative model for personality disorder features. Personality and Mental Health, 12, 107-125.https://doi.org/10.1002/ pmh.1414.

Hopwood, C. J., Kotov, R., Krueger, R. F., et al. (2018). The time has come for dimensional personality disorder diagnosis. Personality and Mental Health, 12, 82-86.

Hopwood, C. J., Wright, A. G., \& Donnellan, M. B. (2011). Evaluating the evidence for the general factor of personality across multiple inventories. Journal of Research in Personality, 45, 468-478. https://doi.org/10.1016/j.jrp.2011. 06.002.

Huprich, S. K. (2015). Introduction: Personality disorders into the 21 st century. In S.K. Huprich (Hrsg.), Personality disorders: toward theoretical and empirical integration in diagnosis and treatment (S. 3-20). Washington: American Psychological Association.

Johansen, M., Karterud, S., Pederson, G., Gude, T., \& Falkum, E. (2004). An investigation oft he prototype validity oft he borderline DSM-IV construct. Acta Psychiatrica Scandinavica, 109, 289-298. https://doi.org/10.1046/j.16000447.2003.00268.x.

Kotov, R., Krueger, R. F., Watson, D., et al. (2017). The hierarchical taxonomy of psychopathology (HiTOP): a dimensional alternative to traditional nosologies. Journal of Abnormal Psychology, 126, 454-477. https://doi.org/10.1037/ abn0000258.

Krueger, R.F. (2013). Personality disorders are the vanguard of the Post-DSM-5.0 era. Personality Disorders: Theory, Research and Treatment, 4, 355-362. https://doi.org/10. $1037 /$ per0000028.

Krueger, R.F., \& Markon, K.E. (2014). The role of the DSM-5 personality trait model in moving toward a quantitative and empirically based approach to classifying personality and psychpathology. Annual Review of Clinical Psychology, 10, 477-501. https://doi.org/10.1146/annurevclinpsy-032813-153732.

Krueger, R. F., Derringer, J., Markon, K. E., Watson, D., \& Skodol, A.E. (2012). Initial construction of a maladaptive personality trait model and inventory for DSM-5. Psychological Medicine, 42, 1879-1890.
Krueger, R. F, Hopwood, C. J., Wright, A. G. C., \& Markon, K. E. (2014). DSM-5 and the path toward empirically based and clinically useful conceptualization of personality and psychopathology. Clinical Psychology: Science and Practice, 21,245-261. https://doi.org/10.1111/cpsp.12073.

Lotfi, M., Bach, B., Amini, M., \& Simonsen, E. (2018). Structure of DSM-5 and ICD-11 personality domains in Iranian community sample. Personality and Mental Health, 12, 155-169. https://doi.org/10.1002/pmh.1409.

Markon, K.E., \& Jonas, K. G. (2015). The role of traits in describing, assessing, and understanding personality pathology. In S. K. Huprich (Hrsg.), Personality disorders: toward theoretical and empirical integration in diagnosis and treatment (S. 63-84). Washington: American Psychological Association.

Miller, D. J., \& Widiger, T.A. (2020). The five-factor model of personality disorders. In C.W. Lejuez \& K. Gratz (Hrsg.), The Cambridge handbook of personality disorders (S. 145-160). Cambridge:Cambridge University Press.

Morey, L.C. (2017). Development and initial evaluation of a self-report form of the DSM-5 level of personality functioning scale. Psychological Assessment, 29, 1302-1308. https://doi.org/10.1037/pas0000450.

Morey, L. C., \& Bender, D. S. (2014). Articulating a core dimension of personality pathology. In J. M. Oldham, A. E. Skodol \& D.S. Bender (Hrsg.), The American textbook of personality disorders (2. Aufl. S. 39-54). Washington: American Psychiatric Publishing.

Ofrat, S., Krueger, R.F., \& Clark, L.A. (2018). Dimensional approaches to personality disorder classification. In W. J. Livesley (Hrsg.), Handbook of personality disorder (2. Aufl. S. 72-87). New York: Guilford.

Olajide, K., Munjiza, J., Moran, P., et al. (2018). Development and psychometric properties of the Standardized Assessment of Severity of Personality Disorder (SASPD). Journal of Personality Disorders, 32, 44-56. https://doi. org/10.1521/pedi_2017_31_285.

Oltmanns, J.R., \& Widiger, T.A. (2018). A self-report measure for the ICD-11 dimensional trait model proposal: the personality inventory for ICD-11. Psychological Assessment, 30,154-169. https://doi.org/10.1037/pas0000459.

Oltmanns, J. R., \& Widiger, T.A. (2020). The five-factor personality inventory for ICD-11: a facet-level assessment of the ICD-11 trait model. Psychological Assessment, 32, 60-71. https://doi.org/10.1037/pas0000763.

Paris, J. (2020). Treatment of borderline personality disorder (2. Aufl.). New York: Guilford.

Pincus, A. L., Dowgwillo, E. A., \& Greenberg, L. S. (2016). Three cases of narcisstic personality disorder through the lens of the DSM-5 alternative model of personality disorders. Practice Innovations, 1, 164-177. https:// doi.org/ 10. 1037/pri0000025.

Pires, R., Sousa Ferreira, A., \& Guedes, D. (2017). The psychometric properties of the Portuguese version of the personality inventory for DSM-5. Scandinavian Journal of Psychology, 58, 468-475. https://doi.org/10.1111/sjop. 12383.

Ringwald, W. R., Edershile, E. A., Woods, W. C., \&Wright, A. G. C. (2020). The need for mechanistic models to translate traits from bench to bedside: commentary. In C.W. Lejuez \& K. Gratz (Hrsg.), The Cambridge handbook of personality disorders (S. 471-474). Cambridge: Cambridge University Press.

Roberts, B.W., Luo, J., Briley, D.A., Chow, P.I., Su, R., \& Hill, P. L. (2017). A systematic review of personality trait change through intervention. Psychological Bulletin, 143, 117-141.https://doi.org/10.1037/bul0000088. 
Samuel, D. B., \& Griffin, S. A. (2015). A critical evaluation of retaining personality categories and types. In S. K. Huprich (Hrsg.), Personality disorders: toward theoretical and empirical integration in diagnosis and treatment (S. 43-62). Washington:American Psychological Association.

Sharp, C., Wright, A. G., Fowler, J. C., Frueh, B. C., Allen, J.G., Oldham, J., \& Clark, L. A. (2015). The structure of personality pathology: Both general (' $\left.\mathrm{g}^{\prime}\right)$ and specific $\left(' \mathrm{~s}^{\prime}\right)$ factors? Journal of Abnormal Psychology, 124, 387-398. https:// doi.org/10.1037/abn0000033.

Skodol, A. E., Bender, D. S., Oldham, J. M., etal. (2011).Proposed changes in personality and personality disorder assessment and diagnosis for DSM-5 Part II: clinical application. Personality Disorders: Theory, Research, and Treatment, 2,23-40. https://doi.org/10.1037/a0021892.

Smith, G.T., Atkinson, E. A., Davis, H.A., Riley, E. N., \& Oltmanns, J. R. (2020). The general factor of psychopathology. Annual Review of Clinical Psychology, 16, 75-98. https:// doi.org/10.1146/annurev-clinpsy-071119-115848.

Somma,A., Krueger, R. F., Markon, K. E., \&Fossati, A. (2019). The replicability of the personality inventory for DSM-5 domain scale factor structure in U.S. and non-U.S. samples: a quantitative review of the published literature. Psychological Assessment, 31, 861-877. https://doi.org/10.1037/ pas0000711.

Tyrer, P., Crawford, M., Mulder, R., etal. (2011). The rationalefor the reclassification of personality disorder in the 11th revision of the International Classification of Diseases (ICD11). Personality and Mental Health, 5, 246-259. https:// doi.org/10.1002/pmh.190.

Tyrer, P., Mulder, R., Kim, Y.-R., \& Crawford, M.J. (2019). The development of the ICD-11 classification of personality disorders: an amalgam of science, pragmatism, and politics. Annual Review of Clinical Psychology, 15, 481-502. https://doi.org/10.1146/annurev-clinpsy050718-095736.

Tyrer, P., Reed, G. M., \& Crawford, M. J. (2015). Classification, assessment, prevalence, and effect of personality disorder. Lancet, 385, 717-726. https://doi.org/10.1016/S01406736(14)61995-4.

Waugh, M. H., Hopwood, C. J., Krueger, R. F., Morey, L. C., Pincus, A. L., \&Wright, A. G. (2017). Psychological assessment with the DSM-5 alternative model for personality disorders: tradition and innovation. Professional Psycholo- gy: Research and Practice, 48, 79-89. https://doi.org/10. 1037 /pro0000071.

Widiger, T.A. (2011).The DSM-5 dimensional model of personality disorder: rationale and empirical support. Journal of Personality Disorders, 25, 222-234. https://doi.org/10. 1521/pedi.2011.25.2.222.

Widiger, T. A., \& Costa, P. T. Jr. (2013). Personality disorders and the five-factor model of personality (3. Aufl.). Washington: American Psychological Association.

Widiger, T.A., \& Trull, T.J. (2007). Plate tectonics in the classification of personality disorder: shifting to a dimensional model. American Psychologist, 62, 71-83. https://doi.org/ 10.1037/0003-066X.62.2.71.

Widiger, T. A., Bach, B., Chmielewski, M., et al. (2019). Criterion A of the AMPD in HiTOP. Journal of Personality Assessment, 101, 345-355. https://doi.org/10.1080/00223891. 2018.1465431.

World Health Organization (1992). International classification of diseases (10. Aufl.). Geneva:WHO.

Wright, A.G., Hopwood, C.J., Skodol, A.E., \& Morey, L.C. (2016). Longitudinal validation of general and specific structural features of personality pathology. Journal of Abnormal Psychology, 125, 1120-1134. https://doi.org/ $10.1037 /$ abn0000165.

Zimmerman, M., Rothschild, L., \& Chelminski, I. (2005). The prevalence of DSM-IV personality disorders in psychiatric outpatients. The American Journal of Psychiatry, 162, 1911-1918. https://doi.org/10.1176/appi.ajp.162.10. 1911.

Zimmermann, J., Böhnke, J. R., Eschstruth, A., Mathews, A., Wenzel, K., \& Leising, D. (2015). The latent structure of personality functioning: Investigating Criterion A from the alternative model for personality disorders in DSM-5. Journal of Abnormal Psychology, 124, 532-548. https:// doi.org/10.1037/abn0000059.

Zimmermann, J., Kerber, A., Rek, K., Hopwood, C. J., \& Krueger, R.F. (2019). A brief but comprehensive review of research on the alternative DSM-5 model for personality disorders. Current Psychiatry Reports, 21, 92. https://doi.org/ 10.1007/s11920-019-1079-z.

Hinweis des Verlags Der Verlag bleibt in Hinblick auf geografische Zuordnungen und Gebietsbezeichnungen in veröffentlichten Karten und Institutsadressen neutral. 